\title{
Diffusion of isobutane in silicalite studied by transition path sampling
}

\author{
Thijs J. H. Vlugt ${ }^{a)}$ \\ Department of Chemical Engineering, University of Amsterdam, Nieuwe Achtergracht 166, \\ 1018 WV Amsterdam, The Netherlands \\ Christoph Dellago \\ Department of Chemistry, University of Rochester, Rochester, New York 14627-0216
}

Berend Smit ${ }^{\text {b) }}$

Department of Chemical Engineering, University of Amsterdam, Nieuwe Achtergracht 166, 1018 WV Amsterdam, The Netherlands

(Received 9 May 2000; accepted 23 August 2000)

\begin{abstract}
The diffusion process of isobutane in the zeolite silicalite has been investigated at $300 \mathrm{~K}$ using transition path sampling. At this temperature, isobutane is preferentially adsorbed at the intersections of silicalite. As the hopping from one intersection to another is a very infrequent event, conventional molecular dynamics techniques cannot be used to compute this hopping rate. Transition path sampling is not affected by this problem and, furthermore, does not require any preconceived notion of the transition mechanism. We use transition path sampling to compute hopping rates between stable states and to locate transition states. For isobutane, we found that not only the position but also the orientation is important in the characterization of the transition state. The Lennard-Jones size parameter $\sigma$ describing the alkane-zeolite interactions has a major influence on both adsorption and diffusion of isobutane in silicalite. (C) 2000 American Institute of Physics. [S0021-9606(00)50943-X]
\end{abstract}

\section{INTRODUCTION}

Detailed knowledge of the adsorption and diffusion behavior of hydrocarbons in zeolites is important in the design of petrochemical applications. ${ }^{1}$ As both adsorption and diffusion experiments can be quite complex and time consuming, the study of the adsorption and diffusion of hydrocarbons in zeolites using computer simulations has been an active area of research. ${ }^{2-12}$ Most of these studies have been focused on the zeolite silicalite. This zeolite has a threedimensional structure of straight channels and zigzag channels that cross at the intersections (see Fig. 1).

From both Monte Carlo (MC) simulations and experiments, it has been found that at low loading, branched alkanes are preferentially adsorbed at the intersections of silicalite. ${ }^{6,13}$ Similar results have been obtained for benzene. ${ }^{14-16}$ These bulky molecules diffuse via a hopping mechanism from one intersection to the next. This hopping, however, is a very infrequent event due to the large free energy barrier between two intersections. ${ }^{17}$ Therefore, conventional molecular dynamics (MD) techniques cannot be used to study this process. A naive way of computing the hopping rate would be to perform MD simulations at higher temperatures, for which MD can be used efficiently. ${ }^{12}$ By assuming that the temperature dependence of the diffusion coefficient is correctly described by the Arrhenius equation one can extrapolate to a lower (desired) temperature. This method will not be able to produce accurate results when the extrapolation is performed over a large temperature range,

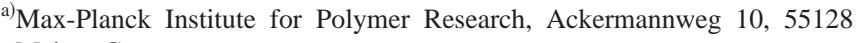
Mainz, Germany

b)Electronic mail: smit@its.chem.uva.nl not only because of extrapolation errors but also because the diffusion mechanism might be different at lower temperatures.

Recently, Smit et al. ${ }^{17}$ have used transition state theory $(\mathrm{TST})^{18,19}$ to calculate the hopping rate of branched alkanes in silicalite; this technique has also been used by Brickmann and co-workers to study the diffusion of xenon ${ }^{20,21}$ and aromatic molecules ${ }^{22,23}$ in the zeolite NaY; the activated diffusion of benzene in $\mathrm{NaY}$ has also been studied by Jousse and Auerbach using this technique. ${ }^{24}$ This method needs a priori information about the possible transition state. Although in principle a dynamical rate calculation is rigorously independent of the dividing surface through which fluxes are computed, such independence is only guaranteed if trajectories can be run for arbitrarily long times. For trajectories of limited duration, dynamical rate calculations can depend sensitively on dividing surface locations; TST thus exhibits the most sensitivity on the dividing surface. The systematic error incurred in such situations can be very difficult to estimate. Therefore, it might be advantageous to use a method in which dividing surface information is not required $a$ priori. This can be done with the transition path sampling method. ${ }^{25}$ It is important to note that if one would be interested in only computing the hopping rate, traditional methods based on TST can be more efficient.

In this paper, we will use transition path sampling to study the hopping of the smallest branched alkane, isobutane, in the zeolite silicalite. In Sec. II, we will briefly summarize this technique. In Sec. III, we will focus on the simulation and model details and their implications, while in Sec. IV we will present our results and a comparison with experimental data. In the Appendix, we will show how to calculate 


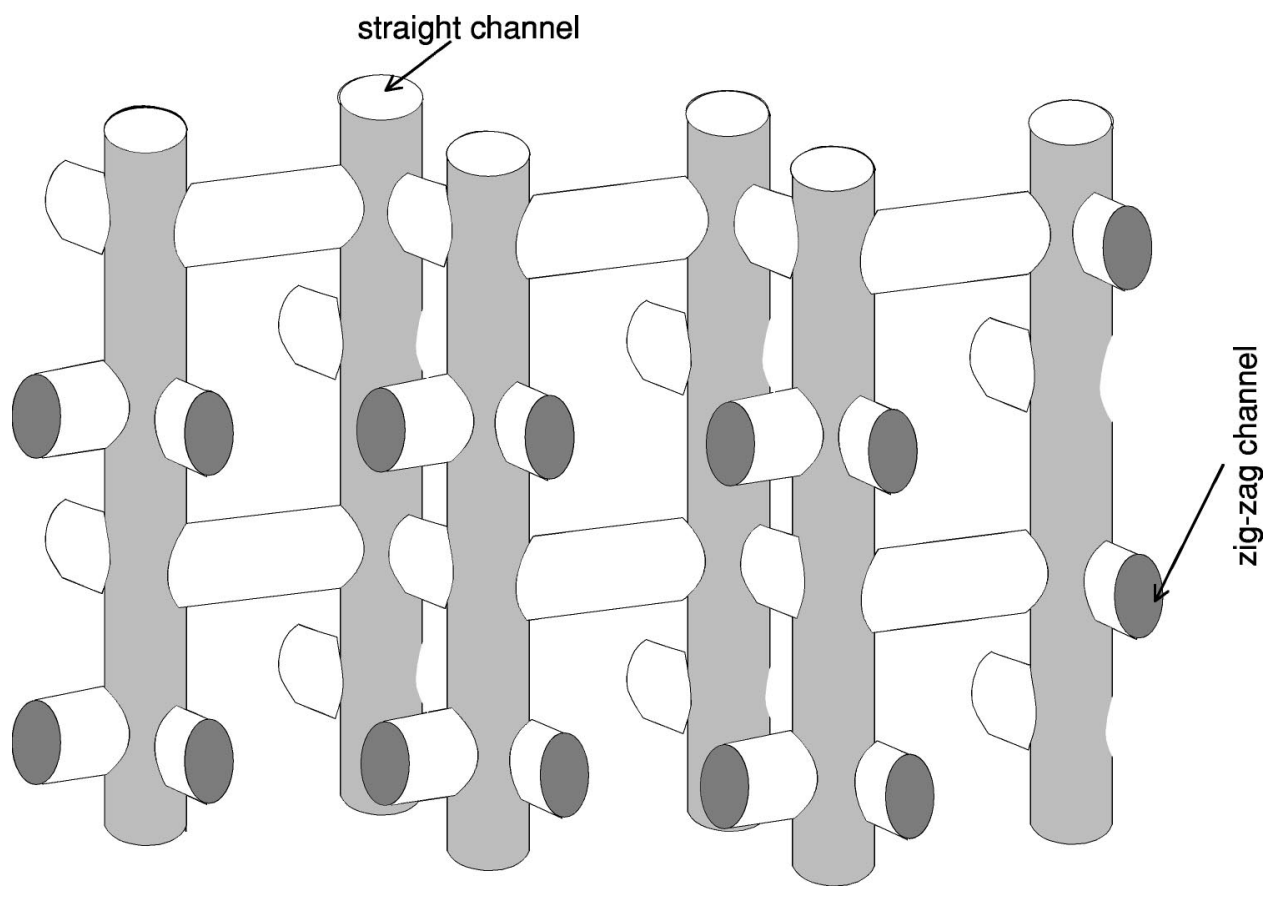

FIG. 1. Schematic representation of the pore structure of silicalite. There are two types of channels: straight and zigzag channels. These channels cross at the intersections.

a free energy profile for a hydrocarbon in a channel of a zeolite.

\section{TRANSITION PATH SAMPLING}

In this section, we will briefly summarize the transition path sampling method for deterministic paths which has been developed by Chandler, Bolhuis, and Dellago. This method is not only able to calculate the hopping rate (and therefore also the diffusion coefficient) between two stable sites (here: the intersections of silicalite) but also to identify the transition states (defined in Sec. II C as the ensemble of points for which the probability to end in each of the stable regions is equal). For a more complete discussion about this simulation technique, the reader is referred to Refs. 25-27.

\section{A. Introduction}

Consider a dynamical system with two stable states, $A$ and $B$, in which transitions from $A$ to $B$ are rare. These stable states could be, for example, intersections of channels in the zeolite silicalite in which a branched alkane is preferentially adsorbed. The transition rate, $k$, from $A$ to $B$ can be calculated from the time derivative of an autocorrelation function $C(t)$,

$$
\begin{aligned}
& k=\frac{d C(t)}{d t}, \quad t_{\mathrm{mol}}<t \ll t_{\mathrm{rxn}}, \\
& C(t)=\frac{\left\langle h_{A}\left(x_{0}\right) h_{B}\left(x_{t}\right)\right\rangle}{\left\langle h_{A}\left(x_{0}\right)\right\rangle},
\end{aligned}
$$

provided that the reaction time $t_{\mathrm{rxn}}$ of the system $[A, B]$ is much larger than the molecular relaxation time $t_{\mathrm{mol}}$ of the system in region $A$ or $B$. As we will see in Sec. III, $k$ is directly related to the diffusion coefficient. In Eq. (2), $x_{t}$ represents the momenta $\mathbf{p}$ and positions $\mathbf{q}$ of the system at time $t$. We will only consider deterministic trajectories for which $x_{t}$ is completely determined by the initial conditions $x_{0}$, i.e., $x_{t}=x_{t}\left(x_{0}\right)$. The functions $h_{A}$ and $h_{B}$ characterize the regions $A$ and $B ; h_{A, B}(x)=1$ when $x \in A, B$, respectively, and $h_{A, B}(x)=0$ otherwise. Note that $A$ and $B$ must be chosen in such a way that $A \cap B=\varnothing$.

Since the function $x_{t}$ is fully determined by the initial condition $x_{0}$, the ensemble averages in Eq. (2) can be written as an integration over the initial conditions weighted with the equilibrium distribution $\mathcal{N}\left(x_{0}\right)$,

$$
C(t)=\frac{\int d x_{0} \mathcal{N}\left(x_{0}\right) h_{A}\left(x_{0}\right) h_{B}\left(x_{t}\left(x_{0}\right)\right)}{\int d x_{0} \mathcal{N}\left(x_{0}\right) h_{A}\left(x_{0}\right)} .
$$

We can also look at this equation as the ensemble average of $h_{B}\left(x_{t}\right)$ weighted with the equilibrium distribution $\mathcal{N}\left(x_{0}\right)$ $\times h_{A}\left(x_{0}\right)$. In other words, $C(t)$ is the fraction of trajectories that start in $A$ with distribution $\mathcal{N}\left(x_{0}\right)$ and reach $B$ after time $t$. Since we are sampling over paths this ensemble is called the path ensemble. A procedure to sample this ensemble would be to perform a MD simulation to generate a new path of length $t$ and subsequently use a MC procedure to decide whether to accept or reject this new path. In this way, we generate an ensemble of paths which we can use to compute ensemble averages. In the remaining part of this paper we will only consider a canonical ensemble of initial conditions $x_{0}$, i.e.,

$$
\mathcal{N}\left(x_{0}\right)=\exp \left[-\beta \mathcal{H}\left(x_{0}\right)\right] .
$$

In principle we could compute $C(t)$ from an "ordinary" path ensemble simulation. This would imply that we generate an ensemble of paths of length $t$ that start at $A$ and we would count all paths that are at time $t$ in $B$. However, since the transition from $A$ to $B$ is a rare event, the number of paths that end in $B$ is so small that such an approach would require very long simulations. Therefore, we need to help the system explore the regions of interest.

Suppose that region $B$ can be defined by the value of an order parameter $\lambda ; x_{t} \in B$ if $\lambda_{\min } \leqslant \lambda\left(x_{t}\right) \leqslant \lambda_{\max }$. For Eq. (2), we may write 


$$
\begin{aligned}
C(t) & =\frac{\int d x_{0} \exp \left[-\beta \mathcal{H}\left(x_{0}\right)\right] h_{A}\left(x_{0}\right) h_{B}\left(x_{t}\left(x_{0}\right)\right)}{\int d x_{0} \exp \left[-\beta \mathcal{H}\left(x_{0}\right)\right] h_{A}\left(x_{0}\right)} \\
& =\int_{\lambda_{\min }}^{\lambda_{\max }} d \lambda P(\lambda, t)
\end{aligned}
$$

in which

$$
\begin{aligned}
P(\lambda, t) & =\frac{\int d x_{0} \exp \left[-\beta \mathcal{H}\left(x_{0}\right)\right] h_{A}\left(x_{0}\right) \delta\left[\lambda-\lambda\left(x_{t}\left(x_{0}\right)\right)\right]}{\int d x_{0} \exp \left[-\beta \mathcal{H}\left(x_{0}\right)\right] h_{A}\left(x_{0}\right)} \\
& =\frac{\int d x_{0} f\left(x_{0}, t\right) \delta\left[\lambda-\lambda\left(x_{t}\left(x_{0}\right)\right)\right]}{\int d x_{0} f\left(x_{0}, t\right)} .
\end{aligned}
$$

$P(\lambda, t)$ can be interpreted as the probability for the system to be in a state with a certain $\lambda$ after time $t$ given that the system is in $A$ at time 0 . Because $P(\lambda, t)$ is quite small in $B$ (i.e., transitions from $A$ to $B$ are rare), special techniques such as umbrella sampling ${ }^{28,29}$ are required to compute $P(\lambda, t)$. As shown in Refs. 26 and 27, it is advantageous to rewrite $C(t)$ as

$$
C(t)=C\left(t^{\prime}\right) \times \frac{\left\langle h_{B}(t)\right\rangle_{F\left(x_{0}, T\right)}}{\left\langle h_{B}\left(t^{\prime}\right)\right\rangle_{F\left(x_{0}, T\right)}},
$$

in which $t, t^{\prime} \in[0, T]$ and

$$
\begin{aligned}
& F\left(x_{0}, T\right)=\exp \left[-\beta \mathcal{H}\left(x_{0}\right)\right] h_{A}\left(x_{0}\right) H_{B}\left(x_{0}, T\right), \\
& H_{B}\left(x_{0}, T\right)=\max _{0 \leqslant t \leqslant T} h_{B}\left(x_{t}\left(x_{0}\right)\right) .
\end{aligned}
$$

The distribution $F\left(x_{0}, T\right)$ can be interpreted as the ensemble of trajectories starting in $A$ and visiting $B$ at least once in the time interval $[0, T]$. In this way, one has to perform only a single transition path sampling calculation of $C\left(t^{\prime}\right)$ when calculating the time derivative of $C(t)$,

$$
k=\frac{d C(t)}{d t}=\frac{C\left(t^{\prime}\right)}{\left\langle h_{B}\left(t^{\prime}\right)\right\rangle_{F\left(x_{0}, T\right)}} \times \frac{d\left[\left\langle h_{B}(t)\right\rangle_{F\left(x_{0}, T\right)}\right]}{d t},
$$

while the functions $\left\langle h_{B}(t)\right\rangle_{F\left(x_{0}, T\right)}$ and $\left\langle h_{B}\left(t^{\prime}\right)\right\rangle_{F\left(x_{0}, T\right)}$ can be calculated from a (single) separate simulation.

\section{B. MC sampling from the distribution $F\left(x_{0}, T\right)$}

In the transition path sampling method, transition pathways are harvested by sampling the path ensemble $F\left(x_{0}, T\right)$ with a MC procedure. ${ }^{26}$ In this subsection, we will present two types of $\mathrm{MC}$ trial moves to generate a new path from an existing one to sample the distribution $F\left(x_{0}, T\right)$. Sampling of the distribution $f\left(x_{0}, t\right)$ is similar. We will use the symbols $n$ and $o$ for the new and old configuration, respectively.

\section{Shooting}

In a shooting move, first one picks a time $t^{\prime}$ randomly from the interval $[0, T]$ and one adds a random displacement to the old positions $\left(\mathbf{q}_{t^{\prime}}(o)\right)$ and old momenta $\left(\mathbf{p}_{t^{\prime}}(o)\right)$,

$$
\mathbf{p}_{t^{\prime}}(n)=\mathbf{p}_{t^{\prime}}(o)+\delta \mathbf{p}, \quad \mathbf{q}_{t^{\prime}}(n)=\mathbf{q}_{t^{\prime}}(o)+\delta \mathbf{q} .
$$

The components $\delta \mathbf{p}$ and $\delta \mathbf{q}$ are chosen at random from a uniform distribution in a finite interval $[-\Delta, \Delta]$. Note that in the original papers of Bolhuis, Chandler, and Dellago ${ }^{26,27}$ only changes in momentum were considered [compare with
Eq. (10)]. However, for systems with large gradients in potential energies (for example, a sorbate in a zeolite) one is able to sample the path ensemble much better when also position changes are applied. This is particularly important at low temperatures for which the contribution of the kinetic energy to the total energy is quite low. Second, one has to construct a new path by integrating backward and forward to obtain $x_{0}(n)$ and $x_{T}(n)$, respectively. To obey detailed balance, the new path has to be accepted with a probability

$$
\begin{aligned}
\operatorname{acc}(o \rightarrow n)= & \min \left(1, \frac{F\left(x_{0}(n), T\right) P_{g}(n \rightarrow o)}{F\left(x_{0}(o), T\right) P_{\mathrm{g}}(o \rightarrow n)}\right) \\
= & h_{A}\left(x_{0}(n)\right) H_{B}\left(x_{0}(n), T\right) \\
& \times \min \left(1, \frac{\exp \left[-\beta \mathcal{H}\left(x_{0}(n)\right)\right] P_{g}(n \rightarrow o)}{\exp \left[-\beta \mathcal{H}\left(x_{0}(o)\right)\right] P_{g}(o \rightarrow n)}\right) .
\end{aligned}
$$

In this equation, $P_{g}(i \rightarrow j)$ is the probability to generate a trial move from state $i$ to state $j$. Since the total energy is conserved along a trajectory, one can compute the second factor on the right-hand side (rhs) of Eq. (11) without integrating the equations of motion. This means that one can reject paths with an unfavorable energy immediately.

For symmetric generation probabilities the terms $P_{g}(o$ $\rightarrow n)$ and $P_{g}(n \rightarrow o)$ cancel. However, one can construct trial-moves for which $P_{g}(o \rightarrow n) \neq P_{g}(n \rightarrow o)$. A well-known example of such a trial-move is configurational-bias Monte Carlo (CBMC) ${ }^{29-33}$. This can, for example, be used to change the orientation of the tail of a long branched molecule like 2-methylpentane, as such a reorientation will not be observed on the time-scales of MD in a narrow channel of a zeolite. One can construct many variations of the shooting trial-move; for example, one can use rotation trial-moves or choose time $t^{\prime}$ with a bias. However, one has to ascertain that the new configuration at time $t^{\prime}$ does not differ too much from the old configuration at $t^{\prime}$ because otherwise the new path will not be a reactive path.

\section{Shifting}

In a shifting move, one translates the initial conditions in time by an amount $\Delta t^{26,27}$

$$
x_{0}(n)=x_{\Delta t}\left(x_{0}(o)\right) \text {. }
$$

To simplify the acceptance rule we choose a symmetric generation probability for $\Delta t$,

$$
P_{g}(\Delta t)=P_{g}(-\Delta t) \text {. }
$$

Due to energy conservation along a trajectory the acceptance rule for this trial-move equals

$$
\operatorname{acc}(o \rightarrow n)=h_{A}\left(x_{0}(n)\right) H_{B}\left(x_{0}(n), T\right) .
$$

Although shifting trial-moves do not sample the phase space ergodically because the energy of the path is not changed, they greatly improve statistics.

\section{Transition state ensemble}

Traditionally, a transition state is defined as a saddle point in the potential energy surface. In complex systems, 
however, saddle points are dense on the potential energy surface and enumeration of saddle points is neither possible nor can it provide insight into the transition mechanism. We therefore adopt a statistical notion of transition states which is equivalent to the procedure described in Ref. 26. We define a configuration $x$ to be a transition state if, for a given kinetic energy $U_{\text {kin }}$, the probability to reach $A$ equals the probability to reach $B$ after a time $\tau$, which is in the order of $t_{\mathrm{mol}}$. This collection of points $\left[x, U_{\mathrm{kin}}\right]$ is called the transition state ensemble. In a transition path ensemble simulation, randomly selected points $\left[x, U_{\text {kin }}\right]$ of several transition paths are analyzed and investigated if they are a transition state. For such a configuration, a large number of MD trajectories with random initial momenta are computed. The fraction of trajectories that reach region $A$ and $B$, respectively, is recorded. When these fractions are equal within certain error bars this configuration is labeled as a transition state. In detail, the following procedure is used:

(1) Select a random point (here: isobutane molecule) of a transition path. For this point $\left[x, U_{\text {kin }}\right]$, we generate $n_{1}$ random momenta from a Maxwell-Boltzmann distribution. These momenta are rescaled in such a way that the total initial kinetic energy of the system $\left(U_{\text {kin }}\right)$ is a constant. The value of $n_{1}$ should be large enough to have some reasonable initial statistics on the probability of reaching regions $A$ and $B$.

(2) For each $n_{1}$ momenta, the equations of motion are integrated. If the system reaches $A$ or $B$, or when the time of the integration exceeds $\tau$, the integration is stopped.

(3) From the $n_{1}$ integrations, we obtain estimates for $p_{A}$ and $p_{B}$, which are, respectively, $\bar{p}_{A}$ and $\bar{p}_{B}$,

$\bar{p}_{A}=\frac{n_{A}}{n_{1}}, \quad \bar{p}_{B}=\frac{n_{B}}{n_{1}}$,

in which $n_{A}, n_{B}$ are the number of paths that reach $A, B$. The errors in these estimates are approximately

$$
\sigma_{A} \approx \sqrt{\frac{\overline{\bar{p}_{A}\left(1-\bar{p}_{A}\right)}}{n_{1}}}, \quad \sigma_{B} \approx \sqrt{\frac{\bar{p}_{B}\left(1-\bar{p}_{B}\right)}{n_{1}}} .
$$

When

$$
\left|\bar{p}_{A}-\bar{p}_{B}\right|>\sigma_{A}+\sigma_{B},
$$

the point $\left[x, U_{\text {kin }}\right]$ is rejected as a transition state. Otherwise, $n_{2}$ additional random momenta are generated and new values for $\bar{p}_{A}, \bar{p}_{B}, \sigma_{A}$, and $\sigma_{B}$ are computed. In case that after $n_{1}+n_{2}$ integrations, $\left|\bar{p}_{A}-\bar{p}_{B}\right|<\sigma_{A}+\sigma_{B}$, the point $\left[x, U_{\text {kin }}\right]$ is accepted as a transition state.

\section{SIMULATION AND MODEL DETAILS}

Isobutane is described with a united-atom model, i.e., $\mathrm{CH}_{3}$ and $\mathrm{CH}$ groups are considered as single interaction centers. ${ }^{34}$ Two bonded united atoms have a harmonic bondstretching potential,

$$
u_{\text {stretch }}(r)=\frac{k_{r}}{2}\left(r-r_{0}\right)^{2},
$$

TABLE I. Lennard-Jones parameters for the zeolite-alkane interactions from different studies: the model proposed by June et al. (Ref. 2), Smit et al. (Ref. 17) and the model developed by us in Refs. 6 and 7. In all

\begin{tabular}{|c|c|c|c|}
\hline & $\frac{\sigma_{\mathrm{CH}_{i} \mathrm{O}}}{[\AA]}$ & $\frac{\epsilon_{\mathrm{CH}_{3} \mathrm{O}} k_{B}}{[K]}$ & $\frac{\epsilon_{\mathrm{CHO}} / k_{B}}{[K]}$ \\
\hline June et al. & 3.364 & 83.8 & 50.0 \\
\hline Smit et al. & 3.64 & 87.5 & 51.3 \\
\hline Vlugt et al. & 3.60 & 80.0 & 58.0 \\
\hline
\end{tabular}
transition path ensemble simulations, we have used a cutoff radius of $10 \AA$ and a truncated and shifted potential.

in which $r_{0}=1.54 \AA$ and $k_{r} / k_{B}=96500 \mathrm{~K} \AA^{-2} .{ }^{35}$ Bondbending between two successive bonds is modeled by a harmonic potential,

$$
u_{\text {bend }}(\theta)=\frac{k_{\theta}}{2}\left(\theta-\theta_{0}\right)^{2},
$$

with $\theta_{0}=114^{\circ}$ and with a force constant equal to $k_{\theta} / k_{B}$ $=62500 \mathrm{~K} \mathrm{rad}^{-2} \cdot{ }^{36}$ For this diffusion study, we have added a dummy hydrogen atom to a $\mathrm{CH}$ group to prevent a branched alkane from inversion; this dummy atom does not have any interaction with the zeolite but it does have a bondstretching potential with the $\mathrm{CH}$ group and bond-bending potentials with the $\mathrm{CH}_{3}$ groups. This unphysical inversion happens at high temperatures or on top of the barrier because the energy barrier for the inversion of a united-atom model of isobutane without the dummy hydrogen atom is much lower than the energy barrier that is in between two intersections. The dummy atom does not affect thermodynamic properties but it can lead to artificial dynamics.

Following Kiselev and co-workers, ${ }^{37}$ the zeolite is modeled as a rigid crystal. This is a quite crude assumption because it has been shown by many authors that the flexibility of the zeolite framework has a large effect on the diffusivity. ${ }^{16,38,39}$ Furthermore, using a rigid zeolite also implies that sorbate molecules cannot exchange energy with the zeolite, which means that the motion of a single sorbate molecule in a zeolite is ballistic. In a transition path ensemble simulation the hopping rate is calculated using a canonical ensemble of initial conditions; this implies that no energy with the zeolite is exchanged during the transition from $A$ to $B$, but that energy can be transferred when the molecules are in the stable states $A$ or $B$. This corresponds to a conventional MD algorithm in which the initial conditions are generated from a Maxwell-Boltzmann distribution. The disadvantage of a flexible zeolite, however, is that those simulations take more than an order of magnitude more CPU time, which is the main reason why we have omitted this flexibility of the framework.

The interactions of the alkane atoms with the zeolite atoms are dominated by the dispersive interactions with the oxygen atoms; ${ }^{37}$ these interactions have been described with a Lennard-Jones potential. We have used the same interactions parameters as used in our previous study on the adsorption of linear and branched alkanes in silicalite; ${ }^{6,7}$ see Table I. The Lennard-Jones size parameter $\sigma_{\mathrm{CH}_{i}-\mathrm{O}}$ is very important for the diffusion mechanism. June et al. ${ }^{2}$ has chosen a 


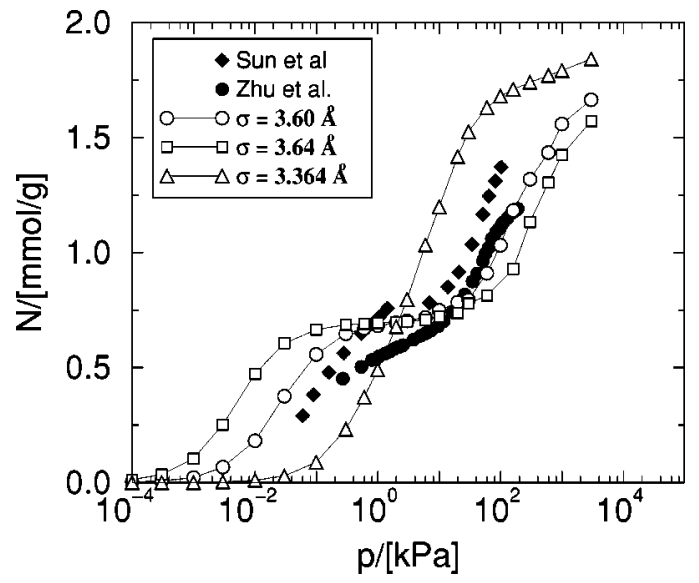

FIG. 2. Adsorption isotherms of isobutane in silicalite. Molecular simulations (open symbols) using the models of Vlugt et al. (Ref. 7) $(\sigma=3.60 \AA$, circles), Smit et al. (Ref. 17) ( $\sigma=3.64 \AA$, squares) and June et al. (Ref. 2) $(\sigma=3.364 \AA$ A triangles). Experimental data (closed symbols) from Zhu et al. (Ref. 13) (circles) and Sun et al. (Ref. 58) (diamonds). See Ref. 7 for details about these simulations. In all simulations, we have used the alkanealkane Lennard-Jones parameters from Table II and the alkane-zeolite interactions from Table I.

rather small value of $\sigma=3.364 \AA$, compared to Smit et al. ${ }^{17}$ and Schuring et al. ${ }^{12}$ who have used $\sigma=3.64 \AA$. The model of June et al. has been used by many authors to study the diffusion of hydrocarbons in silicalite. ${ }^{5,8,9,39,40}$. Recently, we have developed a new force field for linear and branched alkanes in silicalite which uses $\sigma=3.60 \AA \AA^{6,7}$ This force field has been fitted to reproduce experimental data on the heat of adsorption and Henry coefficient of a variety of linear and branched alkanes. In a previous study, we have found that using $\sigma=3.364 \AA$ leads to significantly different adsorption isotherms for $n$-alkanes. ${ }^{7}$ To investigate the reliability of these force fields for isobutane we have computed adsorption isotherms.

In Fig. 2, we have plotted these isotherms for the different models for the alkane-zeolite interactions together with available experimental data. Although the value of $\sigma$ between the models differs by less than $10 \%$, we observe even qualitative differences. The model of June et al. deviates significantly from the other data and does not reproduce the inflection in the isotherm at a loading of four molecules per unit cell (equivalent to approximately $0.7 \mathrm{mmol} / \mathrm{g}$ ), ${ }^{6,41}$ while this inflection is present in both other models and in the two available experimental data sets. As we have shown in
TABLE II. Parameters for the Lennard-Jones potential describing the interactions between pseudo atoms of a branched alkane (Ref. 59). For interactions between different pseudo atoms, the Jorgensen mixing rules have been used (Ref. 60).

\begin{tabular}{lcc}
\hline \hline & $\frac{\epsilon k_{B}}{[K]}$ & $\frac{\sigma}{[\AA]}$ \\
\hline $\mathrm{CH}_{3}-\mathrm{CH}_{3}$ & 98.1 & 3.77 \\
$\mathrm{CH}-\mathrm{CH}$ & 12.0 & 4.10 \\
\hline \hline
\end{tabular}

Ref. 6, the inflection in the adsorption isotherm of isobutane is related to the preferential adsorption of isobutane at the intersections. When all intersections are occupied (at four molecules per unit cell), a large driving force is needed to get additional molecules into the channel interiors. This will result in the inflection in the isotherm. Note that this inflection is also not reproduced using the all-atom consistent valence force field (CVFF) model. ${ }^{10,42}$ Therefore, we will use the parameter set from Ref. 7 that does reproduce the correct sorption thermodynamics in our transition path ensemble simulations.

An important consequence of choosing a value for $\sigma$ that is way too low is that the effective diameter of the channels of silicalite will be much larger. In this way, diffusion of branched alkanes in the framework will not be a rare event anymore and can be studied using conventional MD techniques.

To demonstrate this effect, in Fig. 3 we have plotted the free energy profile of an isobutane molecule along a straight channel and a zigzag channel of silicalite which has been calculated using the CBMC technique. ${ }^{29}$ Details about this type of calculation can be found in Ref. 17 and in the Appendix. To map the coordinates of an isobutane molecule onto a single order parameter $\lambda$, we have defined $\lambda$ in such a way that for a channel between intersections $A$ and $B, \lambda_{A}$ $=0$ and $\lambda_{B}=1$,

$$
\lambda(\mathbf{x})=1-\frac{\left|\mathbf{x}-\mathbf{x}_{B}\right|}{\left|\mathbf{x}_{A}-\mathbf{x}_{B}\right|},
$$

in which $\mathbf{x}_{A}$ and $\mathbf{x}_{B}$ are the centers of the stable regions $A$ and $B$ and $\mathbf{x}$ is the position of the $\mathrm{CH}$ group of isobutane. Indeed, the precise value of $\sigma_{\mathrm{CH}_{i}-\mathrm{O}}$ has a large effect on the height of the barrier. In the model of June et al. the height of the barrier is reduced by almost 50\% compared to the model of Ref. 7. Another important feature is that this free energy
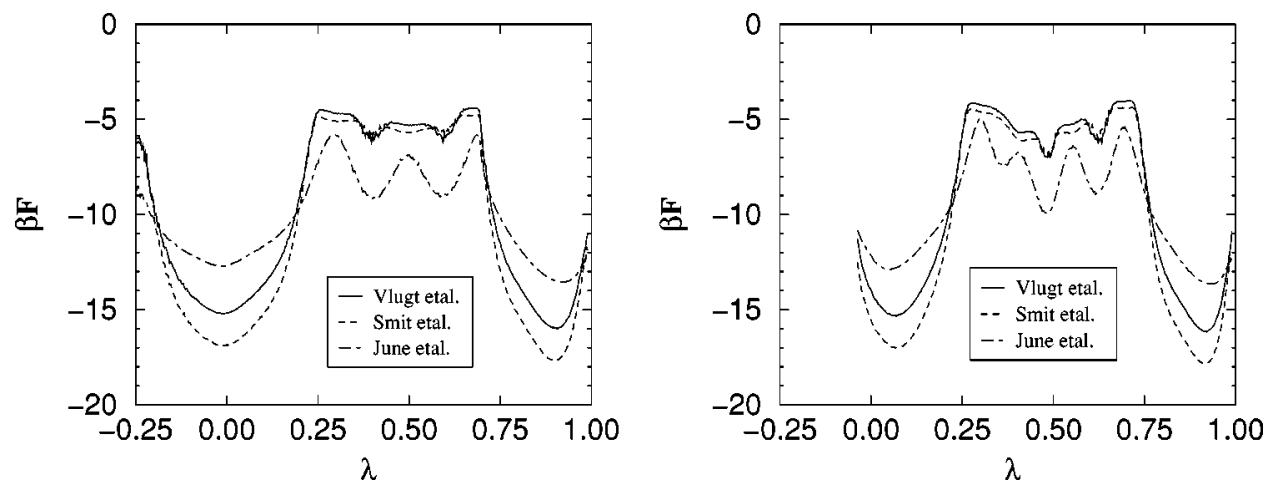

FIG. 3. Free energy of a single isobutane molecule in the straight channel (left) or in the zigzag channel (right) of silicalite as a function of the position in the straight channel for different force fields (Refs. 2, 7, and 17). The two intersections are located at $\lambda$ $=0$ and $\lambda=1$. See the Appendix for details about these calculations. 
TABLE III. Hopping rates (events per second) and diffusion coefficients for diffusion in the straight $(s t)$ and zigzag $(z z)$ channel as computed by transition state theory.

\begin{tabular}{lcccccc}
\hline \hline$\sigma /[\AA]$ & $k_{s t} /\left[\mathrm{s}^{-1}\right]$ & $k_{z z} /\left[\mathrm{s}^{-1}\right]$ & $D_{x x} /\left[\mathrm{m}^{2} / \mathrm{s}\right]$ & $D_{y y} /\left[\mathrm{m}^{2} / \mathrm{s}\right]$ & $D_{z z} /\left[\mathrm{m}^{2} / \mathrm{s}\right]$ & $D /\left[\mathrm{m}^{2} / \mathrm{s}\right]$ \\
\hline 3.364 & $5.1 \times 10^{8}$ & $1.4 \times 10^{8}$ & $1.4 \times 10^{-10}$ & $5.0 \times 10^{-10}$ & $2.0 \times 10^{-10}$ & $2.8 \times 10^{-10}$ \\
3.60 & $1.7 \times 10^{7}$ & $6.4 \times 10^{6}$ & $6.4 \times 10^{-12}$ & $1.7 \times 10^{-11}$ & $8.3 \times 10^{-12}$ & $1.1 \times 10^{-11}$ \\
3.64 & $5.0 \times 10^{6}$ & $1.8 \times 10^{6}$ & $1.8 \times 10^{-12}$ & $5.0 \times 10^{-12}$ & $2.3 \times 10^{-12}$ & $3.0 \times 10^{-12}$ \\
\hline \hline
\end{tabular}

profile shows several local minima and maxima in which isobutane can be stuck when TST with dynamical corrections is used. Transition path sampling suffers less from this problem because the ensemble in which the calculations are performed fixes the endpoint of the trajectory. To compute the hopping rate using TST, we have used the same procedure as in Ref. 17. We have also assumed that the hopping rate is completely determined by the largest free energy barrier. As can be seen in Table III, the hopping rates of the different force fields differ by two orders of magnitude.

In our transition path ensemble simulations, we have used at least $10^{6}$ cycles in every simulation. In every cycle, it is decided at random to do a shooting move in which the momenta are changed (10\%), a shooting move in which isobutane is displaced $(10 \%)$, or a shifting move $(80 \%)$. All maximum displacements were chosen in such a way that $33 \%$ of all trial-moves are accepted. The equations of motion were integrated using a multiple time-step algorithm ${ }^{43,44}$ in which the largest time-step was $10^{-3} \mathrm{ps}$, which results in an average relative deviation of the total energy of around $10^{-5}$. The stable regions $A$ and $B$ were defined as all positions within a distance of $2.5 \AA$ of the center of an intersection of silicalite. A typical calculation of $\left\langle h_{B}(t)\right\rangle_{F\left(x_{0}, T\right)}$ takes 2 weeks on a Linux PC equipped with an AMD K7 (Athlon) $500 \mathrm{MHz}$ processor.

From the hopping rates for the zigzag channel $\left(k_{z z}\right)$ and straight channel $\left(k_{s t}\right)$ one is able to compute the diffusion tensor at zero loading, ${ }^{17,45,46}$

$$
D_{x x}=\frac{1}{4} k_{z z} a^{2}, \quad D_{y y}=\frac{1}{4} k_{s t} b^{2}, \quad D_{z z}=\frac{1}{4} \frac{k_{z z} k_{s t}}{k_{z z}+k_{s t}} c^{2},
$$

in which $a, b$, and $c$ are the unit vectors in the diffusion lattice of silicalite $(a=20.1, b=19.9$, and $c=26.8 \AA$. In the $z$-direction, the diffusion length is two times the length of the unit cell of silicalite). As the total diffusivity $D$ is proportional to the total mean square displacement per unit of time, i.e.,

$$
D=\frac{1}{6} \lim _{t \rightarrow \infty} \frac{\left\langle\mathbf{r}(t)^{2}\right\rangle}{t},
$$

we can write

$$
D=\frac{D_{x x}+D_{y y}+D_{z z}}{3} .
$$

In Eq. (21), we have assumed that two successive hoppings are uncorrelated, which is a reasonable assumption for isobutane but may be invalid for longer 2-methylalkanes because of the orientation of the tail of the molecule. To study the concentration dependence of the diffusion tensor, it would be interesting to use these microscopic hopping rates in a kinetic MC scheme. ${ }^{47,48}$

\section{RESULTS}

\section{A. Calculating the hopping rate}

In Fig. 4, we have plotted the function $\left\langle h_{B}(t)\right\rangle_{F\left(x_{0}, T\right)}$ both for the straight and zigzag channel for $T=15$ ps. For long times, as expected, this function approaches a straight line. In Fig. 5, we have plotted the function $P(\lambda, t)$ for $t$ $=6.0 \mathrm{ps}$ which has been computed by using umbrella sampling. Clearly, this function has several local minima and maxima that are also present in the free energy profiles (see Fig. 3). By integrating over region $B$ we obtain $p_{s t}=6$ $\times 10^{-10}$ and $p_{z z}=8 \times 10^{-10}$.

By combining all results we obtain $k_{s t}=0.4 \times 10^{3}$ and $k_{z z}=0.16 \times 10^{3} \mathrm{~s}^{-1}$. Using Eqs. (21) and (23) we obtain $D_{x x}=2 \times 10^{-16}, D_{y y}=4 \times 10^{-16}, D_{z z}=6 \times 10^{-16}$, and $D$ $=4 \times 10^{-16} \mathrm{~m}^{2} / \mathrm{s}$. Due to the large error bars introduced by
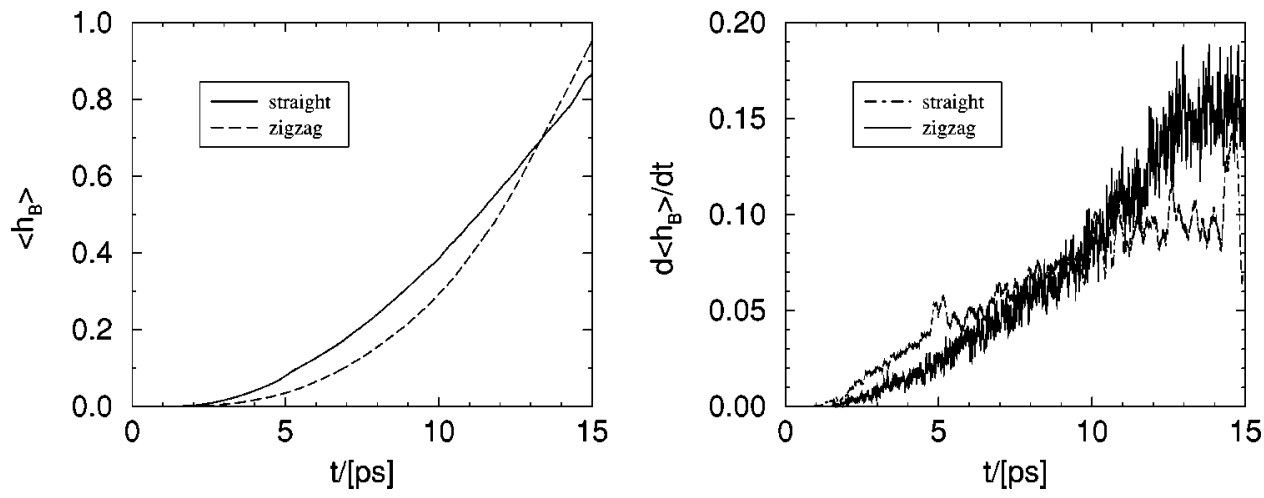

FIG. 4. Left: The function $\left\langle h_{B}(t)\right\rangle_{F\left(x_{0}, T\right)}$ both for diffusion in the straight and zigzag channel. $T=15$ ps. Right: Time derivative of this function. For long times $(t>12 \mathrm{ps})$, a plateau is reached. 


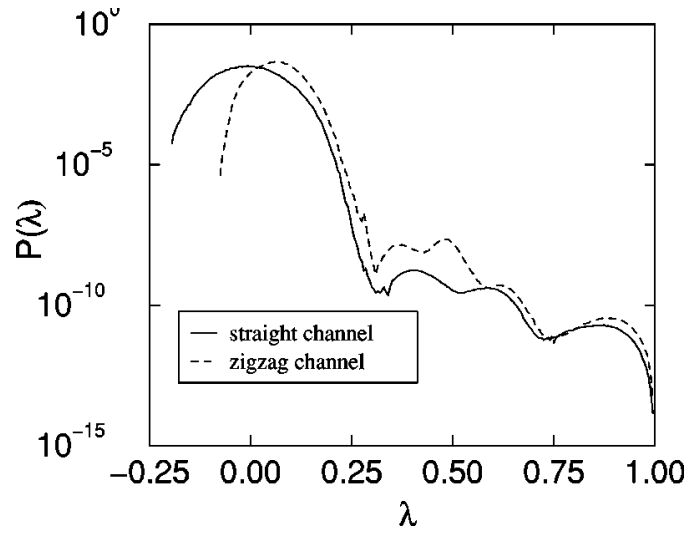

FIG. 5. Probability $P(\lambda)$ to find a particle at position $\lambda$ for $t=6.0 \mathrm{ps}$ for both the straight and zigzag channel. This function has been constructed by matching 10 overlapping windows.

matching the histograms in the free energy calculations we can only say that the computed diffusivity is on the order of $10^{-15}-10^{-16} \mathrm{~m}^{2} / \mathrm{s}$. When we compare this diffusivity with the diffusivity obtained by TST (see Table III) we obtain a transmission coefficient of around $10^{-4}$. The diffusion coefficients are almost equal for the straight and zigzag channel, which can be explained by an almost equal channel diameter of the straight and zigzag channel.

The diffusivity of isobutane in silicalite at $300 \mathrm{~K}$ has been measured by several groups using different experimental techniques; Hufton and Danner (chromatographic) ${ }^{49}$ $2 \times 10^{-12} \mathrm{~m}^{2} / \mathrm{s}$ at $297 \mathrm{~K}$, Shah et al. (membrane permeation $)^{50} 1 \times 10^{-12} \mathrm{~m}^{2} / \mathrm{s}$ at $298 \mathrm{~K}$, Nijhuis et al. (multitrack) ${ }^{51} 6 \times 10^{-13} \mathrm{~m}^{2} / \mathrm{s}$, Bakker et al. (membrane permeation $)^{52} 4 \times 10^{-12} \mathrm{~m}^{2} / \mathrm{s}$, Chiang et al. (chromatographic, extrapolated to $300 \mathrm{~K}$ by using Arrhenius law) ${ }^{53}$ $5 \times 10^{-17} \mathrm{~m}^{2} / \mathrm{s}$, Millot et al. (QENS, extrapolated to $300 \mathrm{~K}$ by using Arrhenius law) ${ }^{54} 2 \times 10^{-13} \mathrm{~m}^{2} / \mathrm{s}$, and Millot et al. (membrane permeation, extrapolated to $300 \mathrm{~K}$ by using Arrhenius law) ${ }^{54} 5 \times 10^{-13} \mathrm{~m}^{2} / \mathrm{s}$. Comparing the experimen- tal results shows that the diffusion coefficients differ by one order of magnitude; the data of Chiang et al. are significantly lower. It is well established in literature that microscopic and macroscopic diffusivities can differ by several orders of magnitude. ${ }^{55}$ Most experimental diffusivities are several orders of magnitude higher than our simulation result.

An explanation for this is that the $\sigma$ value of $3.6 \AA$ we have used is somewhat too high; the simulations of Bouyermaouen and Bellemans ${ }^{39}$ report a diffusivity of $3 \times 10^{-11}$ $\mathrm{m}^{2} / \mathrm{s}$ for MD simulations using $\sigma=3.364 \AA$, which is an order of magnitude larger than most experimental results. For the same system but with a flexible zeolite they found $2 \times 10^{-10} \mathrm{~m}^{2} / \mathrm{s}$. When we insert our TST results for $\sigma$ $=3.364 \AA$ we obtain $2.8 \times 10^{-10} \mathrm{~m}^{2} / \mathrm{s}$, which is an order of magnitude larger that the MD result. As the free energy barrier is largely reduced by using this low value of $\sigma$ we do not expect that their simulations are affected by the inversion of isobutane.

Apparently, the optimal $\sigma$ should be somewhere in between 3.35 and $3.60 \AA$. Also, in our model we have assumed a rigid zeolite lattice to save CPU time, which may result in a diffusivity that is one order of magnitude too low. ${ }^{16,39}$ This suggests that one should carefully choose the model system.

\section{B. Transition state ensemble}

To locate possible transition states, we have used the procedure described in Sec. IIC using $n_{1}=50$ and $n_{2}$ $=150$. Every $10000 \mathrm{MC}$ steps in a simulation of $F\left(x_{0}, T\right)$, 200 randomly selected points of the current path were analyzed by assigning random momenta from a MaxwellBoltzmann distribution and integrating the equations of motion for at most 15000 time-steps. This procedure was continued until 300 transition states have been identified. In Fig. 6, we have plotted the position of the branch for all transition states both for diffusion in the straight channel and in the zigzag channel. Clearly, the transition states are somewhat in between the stable regions $A$ and $B$ (which is not
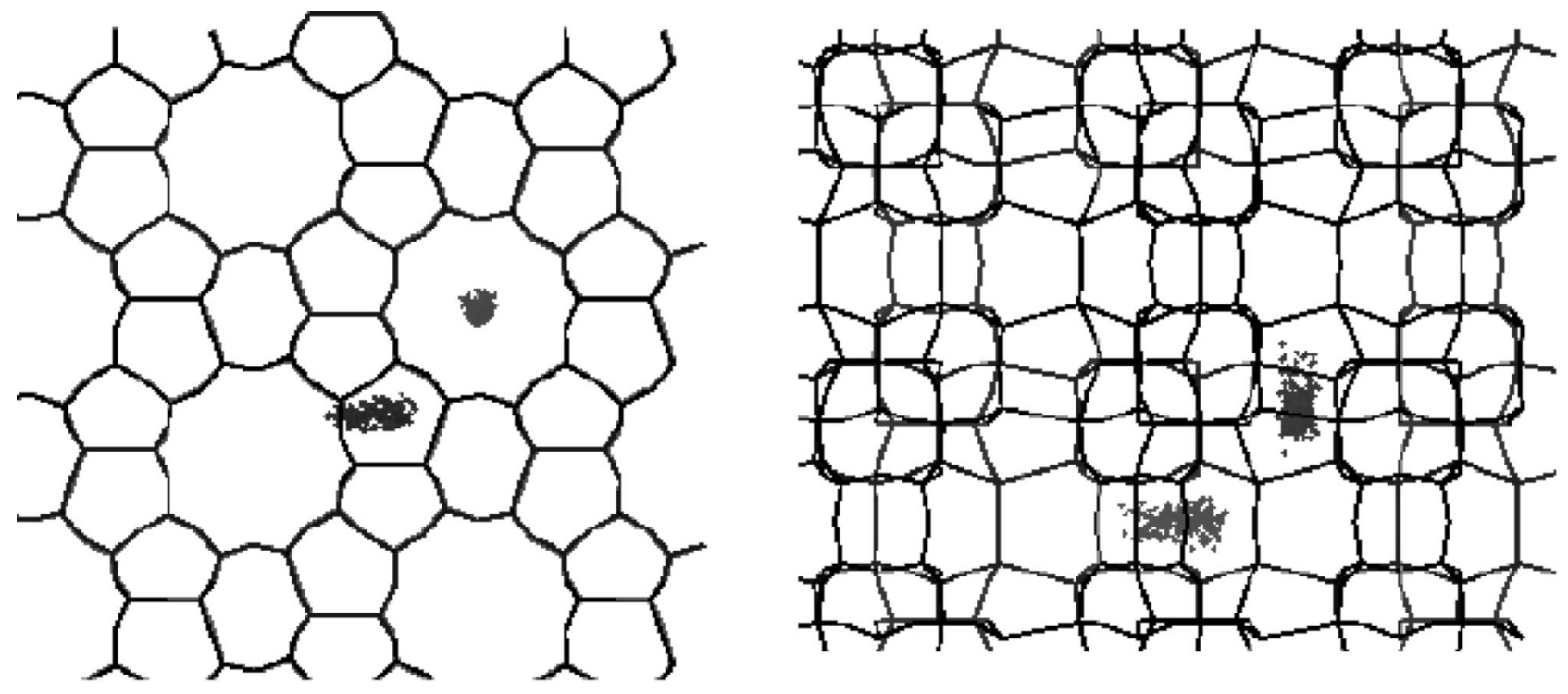

FIG. 6. Schematic representation of all transition states (dots) both for diffusion in the straight channels and in the zigzag channels. Left: Top view, the straight channels are perpendicular to the plane. Right: Side view, the straight channels are from top to bottom while the zigzag channels are from left to right. 


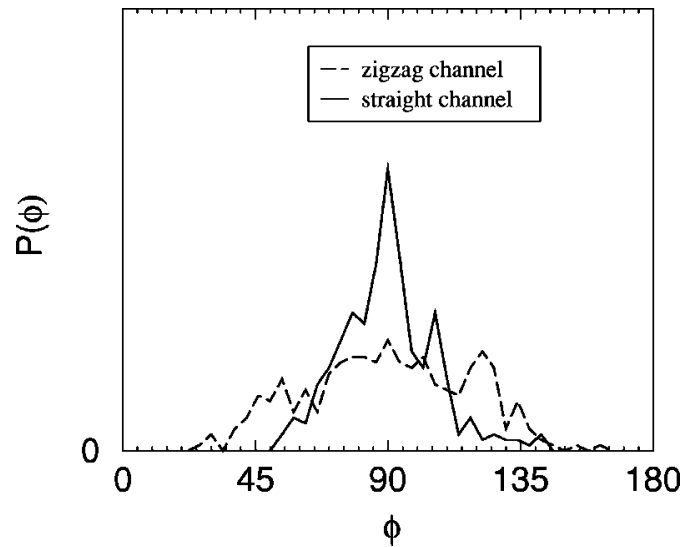

FIG. 7. Probability distribution of the orientational order parameter $\phi$ of all generated transition states for both the straight and zigzag channel.

very surprising of course). As the points are spread over quite a broad part of the channels, there might be another important order parameter involved.

A more interesting property is the orientation of the molecules in the transition state. We have defined the orientational order parameter $\phi$ as the angle between a straight line connecting the centers of the stable regions $A$ and $B$ and the bond vector $\mathrm{C}_{\text {branch }}-\mathrm{H}$ in which $\mathrm{H}$ is a dummy atom that prevents isobutane from inverting itself (see also Sec. III). In Fig. 7, we have plotted the probability distribution of $\phi$. It turns out that the distribution of $\phi$ has a maximum for approximately $90^{\circ}$, which is more pronounced for the straight channel than for the zigzag channel (which is quite obvious because the zigzag channel is not straight). This means that all orientations in which all three methyl-groups all point either to region $A$ or $B$ are not a transition state according to the definition of Sec. IIC. For longer branched alkanes, this suggests that the orientation of the tail will decide to which stable state the molecule will move. This is in agreement with the transition state simulations of Smit et al. ${ }^{17}$ who found that the transmission coefficient of 2-methylhexane in silicalite is extremely low because the orientation of the molecule is not included in the TST calculations.

\section{CONCLUSIONS}

In summary, we have used transition path sampling to study the diffusion of isobutane in silicalite. Our computed diffusion coefficient is significantly lower than the diffusion coefficient obtained by experiments or by the use of transition state theory. The difference with the experimental result might be due to either the use of a rigid zeolite or a LennardJones size parameter describing the alkane-zeolite interactions that is too high. We found that not only the position of the center of mass but also the orientation of an isobutane molecule is important in the identification of transition states. It would be very interesting to compare transition path sampling with the scheme of Ruiz-Montero et al. ${ }^{56}$ This scheme is a modification of transition state theory with dynamical corrections which is suited to study systems with a low trans- mission coefficient efficiently. We would expect that this scheme is able to compute the hopping rate more efficiently than transition path sampling.

\section{ACKNOWLEDGMENTS}

Financial support provided to T.J.H.V. from NWO-CW (Nederlandse Organisatie voor Wetenschappelijk Onderzoek, Chemische Wetenschappen). B.S. acknowledges support from the Dutch Graduate School of Process Technology (OSPT) and NWO-CW through PIONIER. T.J.H.V. would like to thank Marcus G. Martin for useful discussions about alkane-zeolite potentials.

\section{APPENDIX: CALCULATION OF A FREE ENERGY PROFILE}

To compute the hopping rate using transition state theory, one has to know the free energy $(F)$ as a function of the reaction coordinate $(\lambda)$. For a branched alkane, it is convenient to use the position of the $\mathrm{CH}$ group to identify the position of a molecule in a channel, see Eq. (20). To compute the free energy for a given reaction coordinate, we can use CBMC to grow a chain molecule. In this method, a chain molecule of length $N$ is grown step by step. For the insertion of each segment $(j)$, several trial segments $(k)$ are generated and one trial segment $(i)$ is selected with a probability proportional to its Boltzmann factor,

$$
p_{i}=\frac{\exp \left[-\beta u_{i}\right]}{\sum_{i=1}^{i=k} \exp \left[-\beta u_{i}\right]}=\frac{\exp \left[-\beta u_{i}\right]}{w_{j}} .
$$

In Ref. 29 it is shown that the free energy $F(\lambda)$ at a particular position $\lambda$ [here, we have defined $\lambda$ using Eq. (20)] is related to the average Rosenbluth factor $W(\lambda)$,

$$
\exp [-\beta F(\lambda)]=C \times\langle W(\lambda)\rangle, \quad W(\lambda)=\prod_{j=1}^{j=N} w_{j},
$$

in which $C$ is a constant which is determined by the reference frame of the free energy. Note that for branched alkanes, special CBMC techniques are required to compute $W$ correctly. ${ }^{7,57}$

${ }^{1}$ W. Haag, in Studies in Surface Science and Catalysis, edited by J. Weitkamp, H. Karge, H. Pfeifer, and W. Hölderich (Elsevier, Amsterdam, 1994), Vol. 84, pp. 1375-1394.

${ }^{2}$ R. June, A. Bell, and D. Theodorou, J. Phys. Chem. 96, 1051 (1992).

${ }^{3}$ B. Smit and T. Maesen, Nature (London) 374, 42 (1995).

${ }^{4}$ P. Pohl, G. Heffelfinger, and D. Smith, Mol. Phys. 89, 1725 (1996).

${ }^{5}$ R. Runnebaum and E. Maginn, J. Phys. Chem. B 101, 6394 (1997).

${ }^{6}$ T. Vlugt, W. Zhu, F. Kapteijn, J. Moulijn, B. Smit, and R. Krishna, J. Am. Chem. Soc. 120, 5599 (1998).

${ }^{7}$ T. Vlugt, R. Krishna, and B. Smit, J. Phys. Chem. B 103, 1102 (1999).

${ }^{8}$ N. Leonidas and D. Theordorou, J. Phys. Chem. B 103, 3380 (1999).

${ }^{9}$ E. Webb III, G. Grest, and M. Mondello, J. Phys. Chem. B 103, 4949 (1999).

${ }^{10}$ M. Macedonia and E. Maginn, Mol. Phys. 96, 1375 (1999).

${ }^{11}$ M. Macedonia and E. Maginn, Fluid Phase Equilibria 160, 19 (1999).

${ }^{12}$ D. Schuring, A. Jansen, and R. v. Santen, J. Phys. Chem. B 104, 941 (2000).

${ }^{13}$ W. Zhu, J. v. d. Graaf, L. v. d. Broeke, F. Kapteijn, and J. Moulijn, Ind. Eng. Chem. Res. 37, 1934 (1998).

${ }^{14}$ R. Snurr, A. Bell, and D. Theodorou, J. Phys. Chem. 97, 13742 (1993).

${ }^{15}$ R. Snurr, A. Bell, and D. Theodorou, J. Phys. Chem. 98, 5111 (1994).

${ }^{16}$ T. Forrester and W. Smith, J. Chem. Soc., Faraday Trans. 93, 3249 (1997). 
${ }^{17}$ B. Smit, L. Loyens, and W. Verbist, Faraday Discuss. 106, 93 (1997).

${ }^{18}$ D. Chandler, J. Chem. Phys. 68, 2959 (1978).

${ }^{19}$ A. Voter and J. Doll, J. Chem. Phys. 82, 80 (1985).

${ }^{20}$ T. Mosell, G. Schrimpf, C. Hahn, and J. Brickmann, J. Phys. Chem. 100, 4571 (1996).

${ }^{21}$ T. Mosell, G. Schrimpf, and J. Brickmann, J. Phys. Chem. 100, 4582 (1996).

${ }^{22}$ T. Mosell, G. Schrimpf, and J. Brickmann, J. Phys. Chem. B 101, 9476 (1997).

${ }^{23}$ T. Mosell, G. Schrimpf, and J. Brickmann, J. Phys. Chem. B 101, 9485 (1997).

${ }^{24}$ F. Jousse and S. Auerbach, J. Chem. Phys. 107, 9629 (1997).

${ }^{25}$ C. Dellago, P. Bolhuis, F. Csajka, and D. Chandler, J. Chem. Phys. 108, 1964 (1998).

${ }^{26}$ P. Bolhuis, C. Dellago, and D. Chandler, Faraday Discuss. 110, 421 (1998).

${ }^{27}$ C. Dellago, P. Bolhuis, and D. Chandler, J. Chem. Phys. 110, 6617 (1999).

${ }^{28}$ M. Allen and D. Tildesley, Computer Simulation of Liquids (Clarendon, Oxford, 1987).

${ }^{29}$ D. Frenkel and B. Smit, Understanding Molecular Simulations: from Algorithms to Applications (Academic, San Diego, 1996).

${ }^{30}$ J. Siepmann and D. Frenkel, Mol. Phys. 75, 59 (1992).

${ }^{31}$ D. Frenkel, G. Mooij, and B. Smit, J. Phys.: Condens. Matter 4, 3053 (1992).

${ }^{32}$ J. de Pablo, M. Laso, and U. Suter, J. Chem. Phys. 96, 6157 (1992).

${ }^{33}$ M. Falcioni and M. Deem, J. Chem. Phys. 110, 1754 (1999).

${ }^{34}$ J. Ryckaert and A. Bellemans, Faraday Discuss. Chem. Soc. 66, 95 (1978).

${ }^{35}$ S. Nath, F. Escobedo, and J. de Pablo, J. Chem. Phys. 108, 9905 (1998).

${ }^{36}$ P. v. d. Ploeg and H. Berendsen, J. Chem. Phys. 76, 3271 (1982).

${ }^{37}$ A. Bezus, A. Kiselev, A. Lopatkin, and P. Du, J. Chem. Soc., Faraday Trans. 2 74, 367 (1978).

${ }^{38}$ S. Bandyopadhyay and S. Yashonath, J. Phys. Chem. 99, 4286 (1995).
${ }^{39}$ A. Bouyermaouen and A. Bellemans, J. Chem. Phys. 108, 2170 (1998).

${ }^{40}$ E. Maginn, A. Bell, and D. Theodorou, J. Phys. Chem. 99, 2057 (1995).

${ }^{41}$ We would like to thank Marcus G. Martin for pointing this out to us.

${ }^{42}$ A. Hagler, S. Lifson, and P. Dauber, J. Am. Chem. Soc. 101, 5122 (1979).

${ }^{43}$ M. Tuckerman, B. Berne, and G. Martyna, J. Chem. Phys. 97, 1990 (1992).

${ }^{44}$ G. Martyna, M. Tuckerman, D. Tobias, and M. Klein, Mol. Phys. 87, 1117 (1996).

${ }^{45}$ J. Kärger, J. Phys. Chem. 95, 5558 (1991).

${ }^{46}$ F. Jousse, S. Auerbach, and D. Vercauteren, J. Chem. Phys. 112, 1531 (2000).

${ }^{47}$ M. Coppens, A. Bell, and A. Chakraborty, Chem. Eng. Sci. 53, 2053 (1998).

${ }^{48}$ D. Paschek and R. Krishna, Phys. Chem. Chem. Phys. 2, 2389 (2000).

${ }^{49}$ J. Hufton and R. Danner, AIChE J. 39, 962 (1993).

${ }^{50}$ D. Shah, S. Chokchai-acha, and D. Hayhurst, J. Chem. Soc., Faraday Trans. 89, 3161 (1991).

${ }^{51}$ T. Nijhuis, L. v. d. Broeke, M. Linders, J. v. d. Graaf, F. Kapteijn, M. Makkee, and J. Moulijn, Chem. Eng. Sci. 54, 4423 (1999).

${ }^{52}$ W. Bakker, L. v. d. Broeke, F. Kapteijn, and J. Moulijn, AIChE J. 43, 2203 (1997).

${ }^{53}$ A. Chiang, A. Dixon, and Y. Ma, Chem. Eng. Sci. 39, 1461 (1984).

${ }^{54}$ B. Millot, a. Méthivier, H. Jobic, H. Moueddeb, and M. Bée, J. Phys. Chem. B 103, 1096 (1999).

${ }^{55}$ J. Kärger and D. Ruthven, Diffusion in Zeolites and other Microporous Solids (Wiley, New York, 1992).

${ }^{56}$ M. Ruiz-Montero, D. Frenkel, and J. Brey, Mol. Phys. 90, 925 (1997).

${ }^{57}$ M. Martin and J. Siepmann, J. Phys. Chem. B 103, 4508 (1999).

${ }^{58}$ M. Sun, D. Shah, H. Xu, and O. Talu, J. Phys. Chem. 102, 1466 (1998).

${ }^{59}$ J. Siepmann, M. Martin, C. Mundy, and M. Klein, Mol. Phys. 90, 687 (1997).

${ }^{60}$ W. Jorgensen, J. Madura, and C. Swenson, J. Am. Chem. Soc. 106, 6638 (1984). 